\title{
Showing Mutual Support Through Digital Empathy Badges
}

$\begin{array}{ll}\text { Martin de Jode } & \text { Paul Coulton } \\ \text { Andrew Hudson-Smith } & \text { Jonny Huck } \\ \text { Panagiotis Mavros } & \text { Imagination Lancaster, } \\ \text { CASA, } & \text { LICA, } \\ \text { UCL } & \text { Lancaster University, } \\ \text { Gower Street, } & \text { Lancaster, LA1 4YW. } \\ \text { London, WC1E 6BT. } & \text { p.coulton@lancaster.ac.uk } \\ \text { m.dejode@ucl.ac.uk } & \text { j.huck2@lancaster.ac.uk } \\ \text { a.hudson-smith@ucl.ac.uk } & \\ \text { p.mavros.12@ucl.ac.uk } & \text { Jennifer Roberts } \\ & \text { Philip Powell } \\ & \text { InstEAD, } \\ & \text { Department of Economics, } \\ & \text { University of Sheffield, } \\ & 9 \text { Mappin Street, } \\ & \text { Sheffield, S1 4DT. } \\ & \text { j.r.roberts@sheffield.ac.uk } \\ & \text { p.a.powell@sheffield.ac.uk }\end{array}$

\begin{abstract}
Charity badges and empathy (awareness) ribbons are common tokens of support for charities and other worthy causes. In this paper we revisit the concept of smart badges with the aim of developing digital equivalents of the charity badge/empathy ribbon. We describe the design of prototype low-cost digital empathy badges based around infra-red transceiver technology, that light up and play a ringtone in the presence of other badges and we present the findings of a small pilot study involving a dozen badge wearers.
\end{abstract}

\section{Author Keywords}

Smart badge; charity badge; empathy ribbon; IR transceiver;

\section{ACM Classification Keywords}

H.5.m. Information interfaces and presentation (e.g., $\mathrm{HCI}$ ): Miscellaneous.

\section{Introduction}

Cheap charity badges and lapel stickers have for many years been distributed as a token of a donation,

however small. More recently the wearing of Awareness or Empathy ribbons has become fashionable as a symbol of support for various causes. Ribbon wearing originated in the USA during the 1979 Iran hostage crisis with the yellow ribbon. The subsequent adoption 
${ }^{1}$ Lovegety [4] was a relatively cheap ( $\$ 25)$ proximity matchmaking device introduced in Japan in 1998. Produced in male and female versions, the Lovegety used a short-range radio transceiver to locate nearby 'matches' based on a simple user defined profile, producing an audible beep and flashing light when a device of the opposite sex, broadcasting a similar profile was found. of the red ribbon as a symbol of AIDS awareness in the early 1990s led numerous charities to launch ribbon campaigns, with the pink breast cancer awareness ribbon prominent amongst them [5].

In this paper we attempt to extend the concept of the empathy ribbon into the digital sphere with the 'smart' empathy badge. We present the design and early prototypes of cheap digital empathy badges based around infra-red (IR) transceiver (transmitter/receiver) technology that 'light up' in the proximity of another badge, to indicate a shared accord with the cause supported by the badge wearer.

\section{Related Work}

There has been considerable work in the area of wearable computing devices in a badge-like form factor. One of the early prototypes was the Active Badge location system developed by Olivetti Research [6], which used IR LED-enabled badges to determine the location of staff relative to static IR sensors in work places such as hospitals. Other work has focused on computer-supported cooperative working (CSCW) particularly in the context of smart name badges aimed at conference delegates. Workers at MIT Media Labs have trialled various prototype computational badges based around microcontrollers and IR transceiver technology in conference scenarios and corporate events. In the 'Thinking Tag' project [1] delegates preprogrammed their smart name badge with their responses to a set of five questions (agree, disagree). Each badge had five bicoloured LEDs that, upon encountering another badge wearing delegate, lit up in accordance with the extent to which they agreed or disagreed on the answers to the questions. Building on this theme MIT went on to develop Meme Tags [2] that allow delegates to electronically share memes (ideas or opinions) displayed on a LCD display mounted on the badge. More recently increasingly sophisticated devices have been reported including the Sociometer [3] featuring a range of sensors including an IR transceiver, a microphone and accelerometers to record and explore the network dynamics of social gatherings.

Inevitably Smart badges have also been used in dating and match making scenarios, see for example the Lovegety device ${ }^{1}$. More recently the ubiquity of the smartphone has superseded custom devices as the platform of choice for many group awareness applications, particularly those related to dating.

\section{Design Considerations}

The primary design constraint was to keep the bill of materials to below $\$ 5$, so that the badge could be a token of relatively small donations. Being a wearable device the other principal constraints were size (small and light like a badge) and battery life (as long as possible, but at least several days). In light of the above and in common with previous workers we chose IR technology similar to that used in TV remote controls as the proximity detecting method, as it has the advantages of low cost and low power consumption due to its maturity and mass production. Various IR LEDs and IR receiver chips were tested, with the Osram SFH 4547 IR LED and the Vishay TSOP4840 receiver being typical of the types employed. For processing we chose the Picaxe 08M2 microcontroller, which is cheap and easy to program as well as featuring in-built support for decoding the Sony IR remote control protocol. The Picaxe microcontroller also provides the ability to drive audio transducers to play tunes programmed as ringtones via the in-built 'tune' function. A piezoelectric 


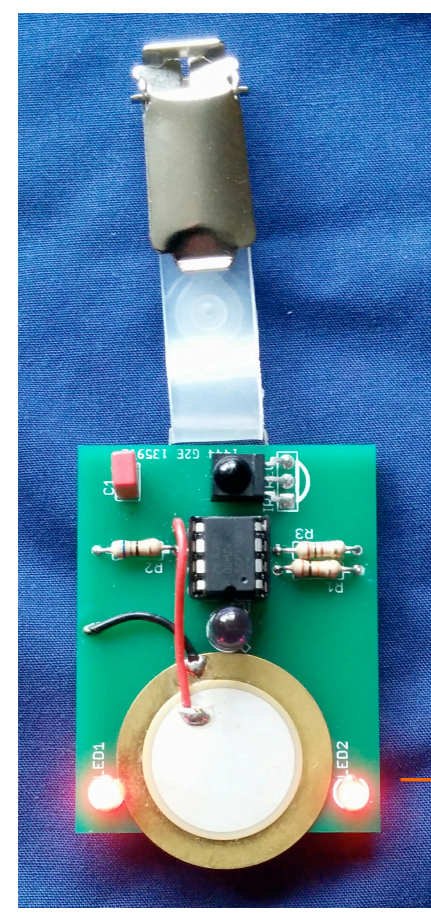

disc was used to provide a low-cost 'speaker. A single 3 Volt CR2032 coin cell powers the badge. Upon encountering another badge (detecting the IR protocol emitted by a fellow badge wearer) visible LEDs mounted on the badge light up (Figure 1) and the ringtone plays. The playing of the ringtone provides the wearer with additional notification of an encounter with a fellow badge wearer, which is particularly useful in bright ambient conditions (e.g. sunlight). To avoid the irritation of the ringtone continually playing during prolonged interactions a delay was introduced into the microcontroller software to prevent replay until after a set time interval had elapsed, programmed to be about 5 minutes. It is easy to customise sets of badges for a particular cause or community via the choice of ringtone and remote control channel (the Sony protocol supports 128 distinct channels).

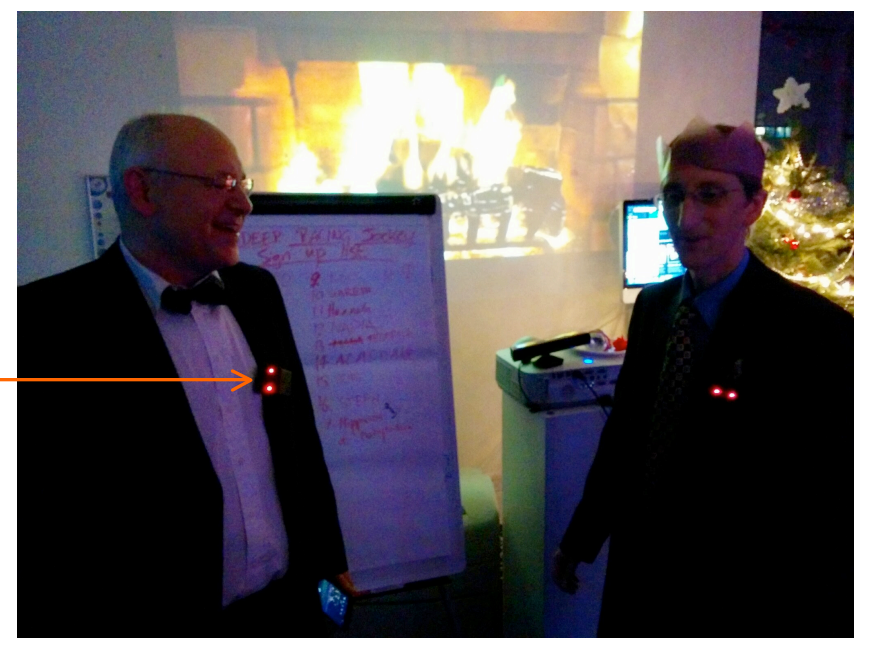

Figure 1. Initial pilot study of the empathy badges at the departmental Christmas party showing two badges interacting.

\section{Initial Evaluation}

The empathy badges are 'line of sight' devices as a consequence of the IR transceiver technology. The IR LED broadcasts continuously and so to extend battery life the IR LED drive current was resistor-limited to a mean (RMS) current of about $0.2 \mathrm{~mA}$, giving a total quiescent (non-interacting) current draw for the badge of less than $1 \mathrm{~mA}$. This gave interaction distances of about 3-5 meters and a battery life of around 150 hours, depending on the number and duration of interactions.

We performed a small pilot study at a departmental Christmas party employing a dozen prototype badges. The technology worked well and received favourable responses. For example one user commented: "It was kind of weird when me, A and B were standing together and all the badges were lighting up". Another user added: "There were occasions when I was walking around and it would light up and I suddenly became more aware of who was around me, who I had connected with". The smart badges encouraged wearers to interact with people that they otherwise may not have, with one wearer commenting, "I talked to all fellow badge wearers including people I didn't previously know". One participant suggested arranging the visible LEDs in the shape of an empathy ribbon. This led to a further design iteration (Figure 2) using more compact surface mount components, which also allowed us to reduce the footprint of the $\mathrm{PCB}$, allowing the use of transparent plastic memory card cases as cheap enclosures with good IR transmission

characteristics. The case also provided the resonant enclosure for the piezoelectric transducer, which when mounted on the internal face provided increased volume compared with direct mounting on the PCB. 


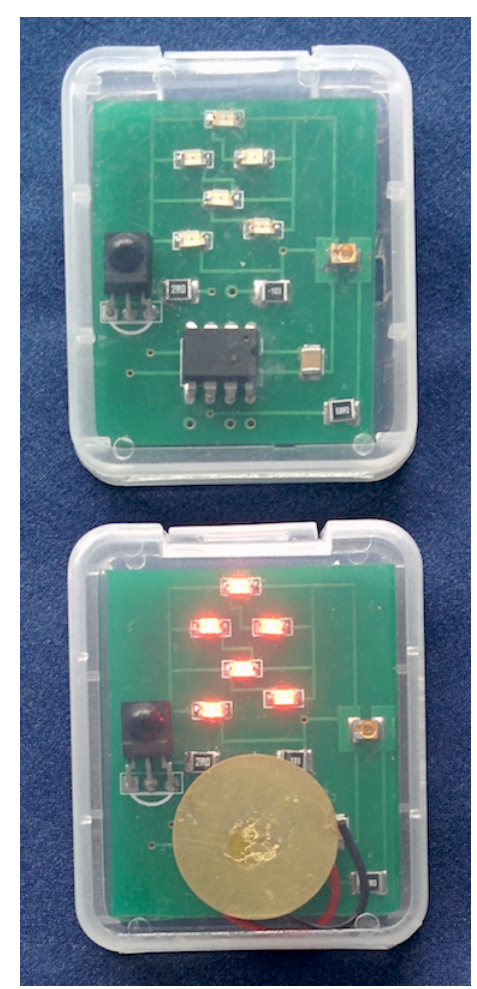

Figure 2 Later prototype empathy badges using surface mount components and a

Compact Flash memory card case for the enclosure. Note the piezo disc mounted internally on the lower badge. These prototypes measured approximately $5 \times 4 \mathrm{~cm}$ and weighed 14 grams including the battery.

\section{Discussion and Further Work}

Although smart badges incorporating IR transceivers have been reported previously in a number of contexts, the idea of using low cost, almost disposable, smart badges analogously to empathy ribbons and charity badges does not seem to be have been previously explored. Purely in terms of technology, as distinct from purpose, our empathy badges have much in common with the earlier Thinking Tag [1], with the addition of ringtone functionality. However, due to commoditization of the technology involved, these badges can now be produced at a much lower cost ( $\$ 5$ $v$ Thinking Tag $\sim \$ 20$ in 1996) with greater battery life (150 hours from one coin cell $v 50$ hours from 2 coin cells) opening up the novel digital empathy ribbon/charity badge usage.

In a detailed survey of traditional ribbon wearers Moore [5] found that a significant proportion could not remember which cause their ribbon represented and

\section{Acknowledgements}

We thank all the volunteers who helped in our evaluation. The authors gratefully acknowledge support from the EPSRC under grant EP/L003635/1.

\section{References}

[1] Borovoy, R., McDonald, M., Martin, F. and Resnick, $M$. Things that blink: computationally augmented name tags. IBM Syst. J. 35, 3-4 (1996), 488-495.

[2] Borovoy R., Martin, F., Vemuri, S., Resnick, M., Silverman, B. and Hancock. C. Meme tags and community mirrors: moving from conferences to collaboration. In Proc. CSCW '98, ACM Press (1998), 159-168. that at times the ribbon appeared to be "more akin to a fashion accessory than a charity token". Hence taking a fresh approach to such charity tokens may be timely. By 'lighting up' in the presence of another wearer we feel these smart empathy badges help create stronger connections between fellow wearers than traditional charity tokens, which can lead to conversation and increased mutual understanding, support and empathy with the cause promoted by the token and between the individual wearers themselves. These smart badges also have obvious potential for mutual support groups such as, for example, Alcoholics Anonymous. The ability of the empathy badges to create connections was demonstrated by our initial evaluation where wearers admitted to talking to others wearers who they did not previously know and otherwise would have been unlikely to chat to. The current prototypes have so far been produced in small quantities (a couple of dozen) but we intend to explore scaling up production, which can then be employed in larger scale evaluations.

[3] Choudhury, T. and Pentland, A. Sensing and Modeling Human Networks using the Sociometer. In Proc. ISWC '03, IEEE Computer Society, (2003), 216222.

[4] Lovegety, http://en.wikipedia.org/wiki/Lovegety (retrieved 04/30/2015).

[5] Moore, S. E. H. Ribbon Culture: Charity, Compassion and Public Awareness. Palgrave Macmillan, London, UK, 2008.

[6] Want, R., Hopper, A., Falcão, V. and Gibbons, J. The active badge location system. ACM Trans. Inf. Syst. 10, 1 (1992), 91-102. 